VEREINIGT M IT FOLIA HAEMATOLOGICA, NEUE FOLGE ZEITSCHRIFT FÜR DIE GESAMTE BLUTFORSCHUNG Organ der Deutschen Gesellschaft für Hämatologie Organ der Deutschen Gesellschaft für Bluttransfusion und Immunhämatologie

\title{
LEITARTIKEL
}

Medizinische Klinik und Poliklinik

(Direktor: Prof. Dr. W. H. Hauss)

der Westfälischen Wilhelms-Universität Münster

\section{Impulszytophotometrie in der Hämatologie}

Thomas Büchnet

In dieser noch etwas frühen Bestandsaufnahme ist zu fragen, welche begründeten Erwartungen und welche bereits vorliegenden Ergebnisse das Interesse an der Impulszytophotometrie (Dittrich und Göbde 1969 [7], Göbde 1972 [8]) in der Hämatologie rechtfertigen können.

Erwartungen liegen darin, daß 1. der DNA-Gehalt der Zelle ein relevanter Wert ist, der den Zustand während der Proliferation oder eine Aneuploidie anzeigt, 2. die Messung an der einzelnen Zelle genau genug ist, 3. statistische Fehler durch reichliche Stichproben wegfallen und 4. die Handhabung den Anforderungen der klinischen Routine genügt.

Begründet erscheinen diese Erwartungen, indem

1. DNA-Werte einzelne Stadien der Proliferation wie die S-Phase und die $G_{2}-P h a s e$ oder die Mitose eindeutig markieren bzw. Aneuploidie auch an sich nicht teilenden Zellen, deren Karyotyp unzugänglich ist, wiedergeben,

2. die Streuung der Meßwerte etwa für Zellen mit einfachem DNA-Gehalt ( $\mathbf{G}_{1-}$ Zellen) bei $\pm 3,4$ bis 6,5\% des Mittelwertes liegt (Bücbner et al. 1973 [4]), so daß eine deutliche Trennung des $G_{1}$-Anteils, des S-Phase-Anteils und des $\left(G_{2}+\right.$ Mitosen)-Anteils möglich ist.

3. 10000 bis 100000 Zellen pro Histogramm gemessen werden, dadurch ideale Normalverteilungen für den $G_{1^{-}}$und den $G_{2}$-Anteil und ein glattes Plateau für den S-Anteil erreicht werden und

4. einfachste Präparation der Proben (Büchner et al. 1973 [5]) und hohe Meßrate (bis 1000 Zellen/sec) ausgeschriebene Histogramme innerhalb $1 \mathrm{~h}$ liefern.

Somit sind auch Erwartungen von Aufschlüssen über die Proliferation der Zellkollektive im Knochenmark und Blut in vivo, über aneuploide Zellinien und über durch Therapie geschädigte und in bestimmten Phasen arretierte Zellen begründet.

Eingegangen am 10. 8. 1973. 
Das Impulszytophotometer (ICP 11 von Phywe-Göttingen), von Dittrich und Göbde (1969 [7]) entwickelt, mißt fluorochromierte Zellinhaltsstoffe im UV-Licht beim Durchfluß einer Zellsuspension anhand der Fluoreszenzimpulse, registriert und klassifiziert die Einzelwerte elektronisch und schreibt sie als Verteilungskurve der

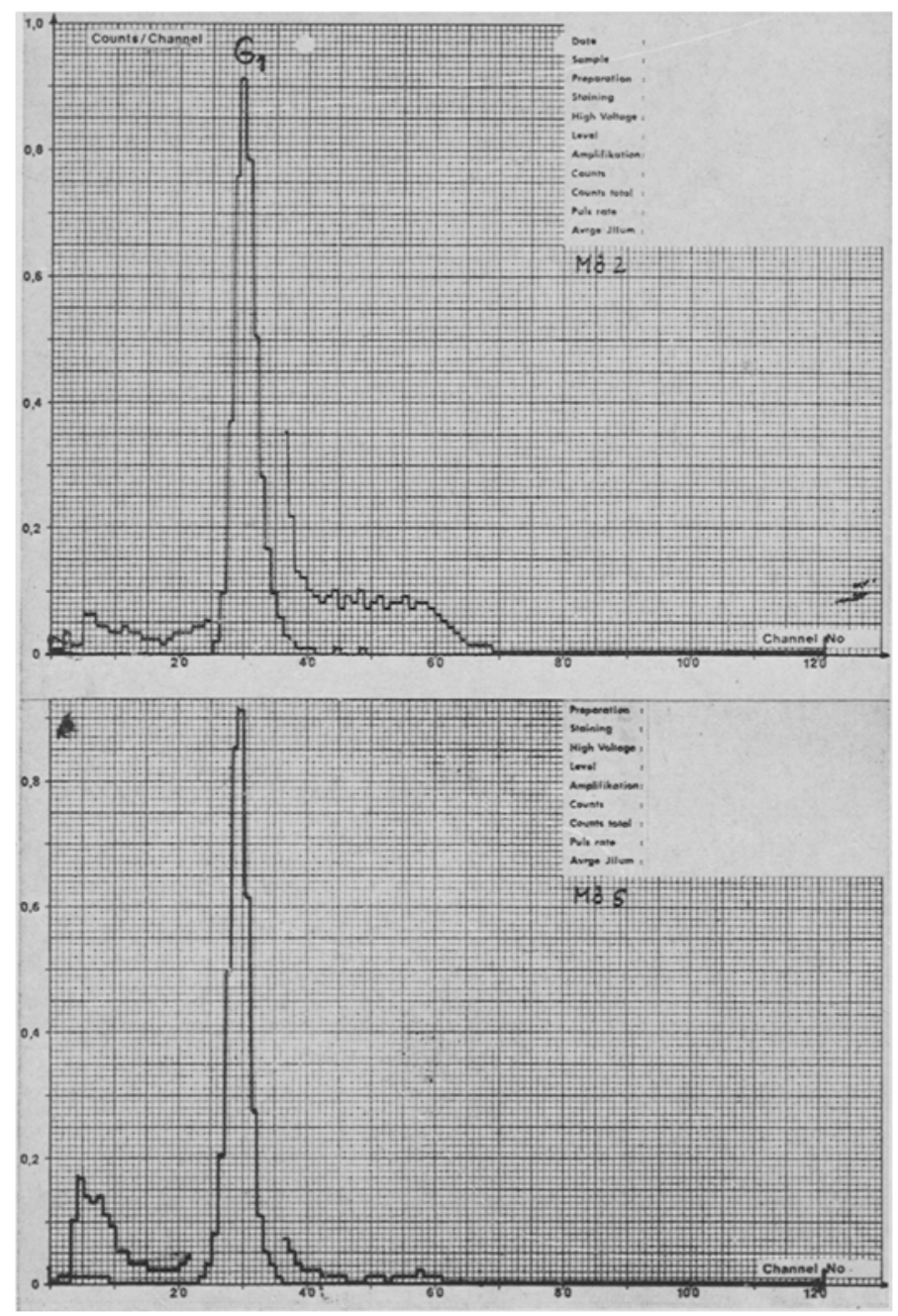

Abb. 1: DNA-Histogramm des peripheren Blutes eines 35 Jahre alten Patienten mit wahrscheinlichem Virusinfekt ohne typischen serologischen Befund, mit Fieber und multiplen Lymphomen.

Oben, bei Klinikaufnahme: 29000 Leukozyten $/ \mathrm{mm}^{3}$, 90\% Lymphozyten, darunter zahlreiche Reizformen. Die Kurve ergibt 5\% Zellen mit DNA-Werten im Bereich der S-Phase (rechts vom $G_{1}-G i p f e l$ ). Links und rechts vom $G_{1}-G i p f e l$ wurde die Kurve zusätzlich mit 10facher Verstärkung registriert.

Unten, 13 Tage nach Aufnahme: 9600 Leukozyten $/ \mathrm{mm}^{3}$, 78\% Lymphozyten. Rückgang der S-Phase-Zellen auf unter 1\%. Der kleine Anteil von höheren DNA-Werten entspricht der normalen Koinzidenzrate von etwa $1 \%$. 
Zellzahl pro Meßwert aus. Für die DNA-Messung wurde von den Urhebern Ethidiumbromid, das doppelsträngige Nukleinsäuren quantitativ anfärbt (LePecq und Paoletti 1967 [12]) eingeführt (Dittrich und Göbde 1969 [7]).

Seit 1970 wurden in Zusammenarbeit mit den Urhebern der Methode Erfahrungen in der Hämatologie mit dem ICP gewonnen (Büchner et al. 1971 [1,2]). Dabei wurde auch eine einfache Methode der Zellpräparation für Blut und Knochenmark erarbeitet (Bücbner et al. 1973 [5]), wobei Erythrozyten durch Hämolyse in Wasser beseitigt werden und die Suspension in 35\% Äthanol konserviert wird, während enzymatische Behandlung, Leukozytenanreicherung und Zentrifugation entfallen, so daß präparative Veränderungen der Kurvenform, Selektivierung bestimmter Zellarten und Zellverklebungen vermieden werden.

Unter diesen Voraussetzungen erlaubt das DNA-Histogramm eine anteilmäßige Bestimmung der $G_{1}\left(+G_{0}\right)$-Zellen und der $\left(G_{2}+M\right)$-Zellen durch Planimetrie sowie der S-Phase-Zellen anhand des Produkts aus $\left(G_{1}-G_{2}\right)$-Abstand und Kurvenhöhe zwischen $G_{1}$ und $G_{2}$ (nach Göbde 1973 [9]).

Einige typische Befunde sind im folgenden anhand von Fotos der Originalhistogramme wiedergegeberi.

Die Resultate zeigten zunächst, daß Informationen über Zellpopulationen, wie sie mit aufwendigen Techniken zu erhalten sind, mittels ICP leichter gewonnen werden können. So ergaben sich die quantitativen Anteile von proliferierenden Zellen mit erhöhtem DNA-Gehalt im Knochenmark und Blut beim Blutgesunden, bei Perniciosa und verschiedenen Leukämien (Bïchner et al. 1971 [1,2]). Es zeigt sich etwa bei Virusinfekten im Blut ein höherer Anteil von Zellen im Bereich der S-Phase, der mit

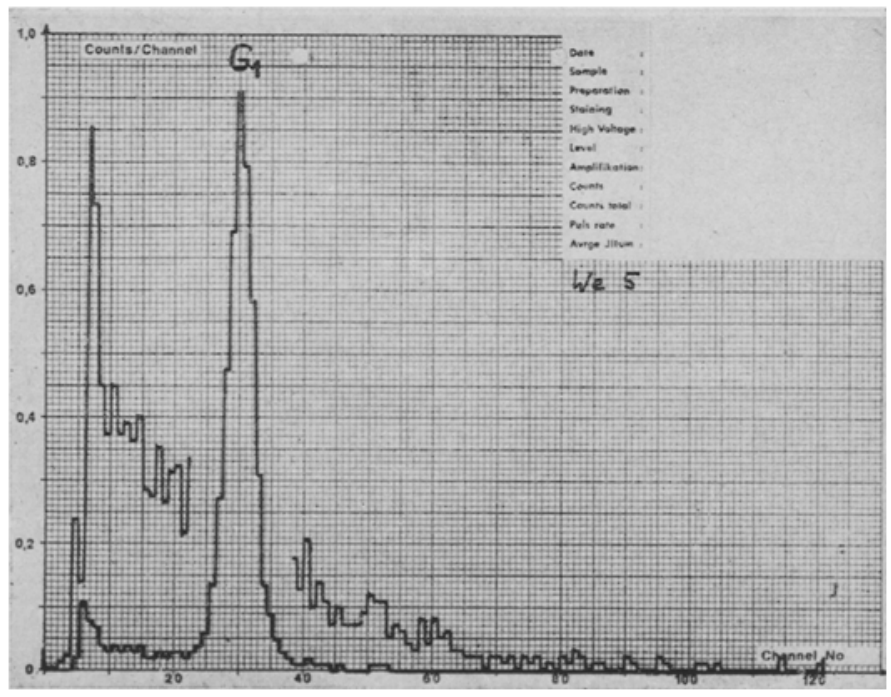

Abb. 2: DNA-Histogramm mit vermehrten Zellfragmenten auf Grund erhöhter Lädierbarkeit der Zellen im peripheren Blut bei AML nach zytostatischer Therapie durch 5tägige Infusion von Cytosin-arabinosid ( $3 \mathrm{mg} / \mathrm{kg} / 24 \mathrm{~h}$ ). Die $10 \mathrm{fach}$ überhöhte Kurve zeigt die typische Exponentialfunktion mit Kurvenanstieg gegen Null. Die Werte rechts von $G_{1}$ entstanden durch Verklebung der Fragmente untereinander. 


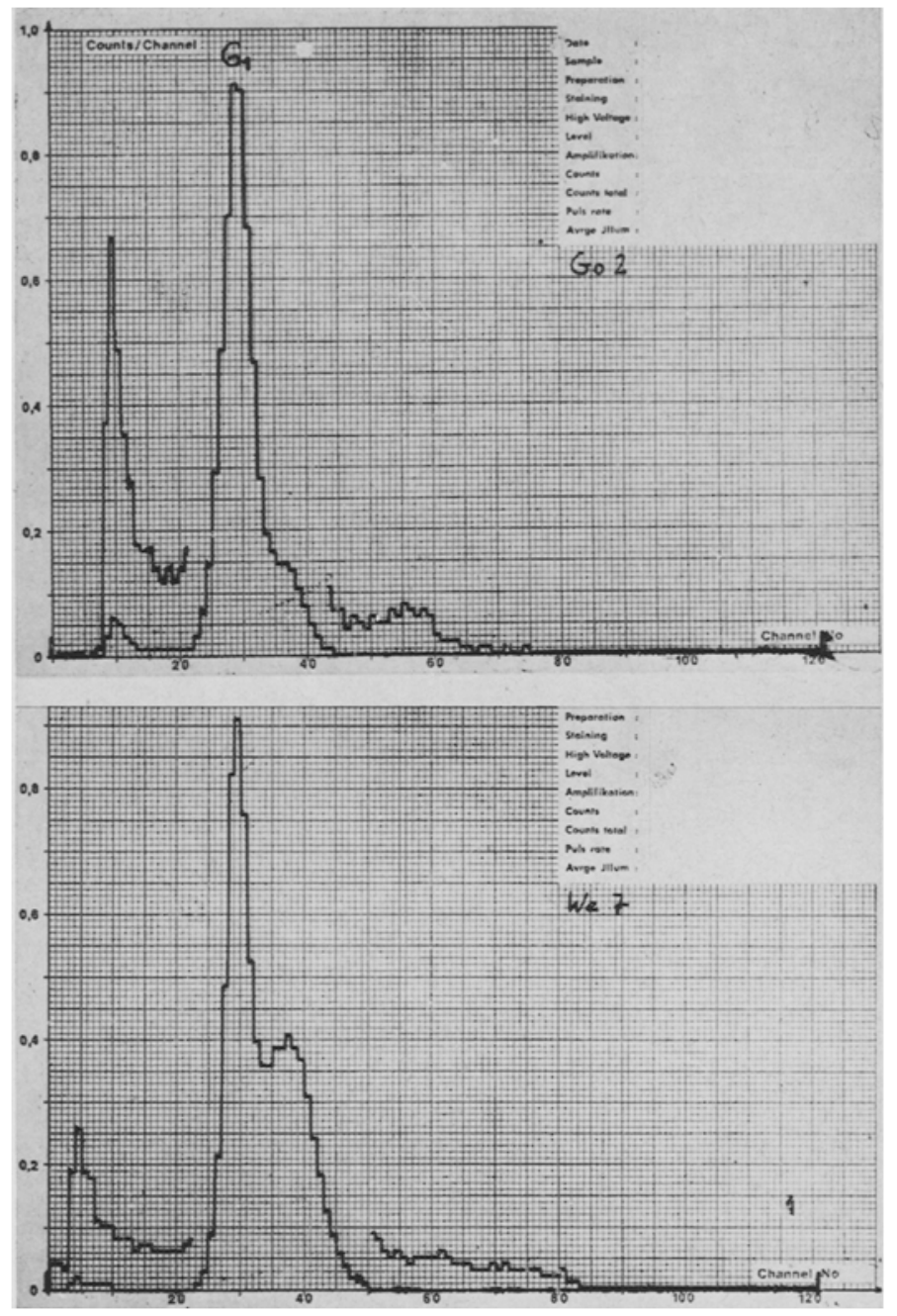

Abb. 3: Oben. Anhäufung von Zellen aus dem Blut im Bereich der frühen S-Phase (rechts neben dem $G_{1}-$ Gipfel) nach 48stündiger Infusion von Cytosin-arabinosid bei AML. Der linke Gipfel zeigt vermehrte Zellfragmente.

Unten. ausgeprägte Anhäufung mit DNA-Werten im Bereich dex frühen S-Phase im Blut (Extragipfel rechts vom $G_{1}$-Gipfel) $40 \mathrm{~h}$ nach Injektion von Adriamycin (1 mg/kg) bei AML.

Abb. 4: Oben. DNA-Histogramm von Blutzellen eines Patienten mit AML vor Therapie. Mitte, 19 Tage nach 4wöchiger Gabe von Dauno-Rubidomycin + Vincristin (wöchentlich je $1 \mathrm{mg} / \mathrm{kg}$ Ondena $+0,025 \mathrm{mg} / \mathrm{kg}$ Vincristin). Zunahme von $\mathrm{G}_{2}-$ Zellen (2. Gipfel, Mitosen fehlten morphologisch) und Zellen im Bereich der späten S-Phase (Asymmetrie des 2. Gipfels nach links).

Unten. 24 Tage nach Therapie mäßiger Rückgang $\operatorname{der} \mathrm{G}_{2}$-Zellen-Anhäufung, keine Vermehrung von Zellen im Bereich der späten S-Phase mehr (2. Gipfel symmetrisch). 


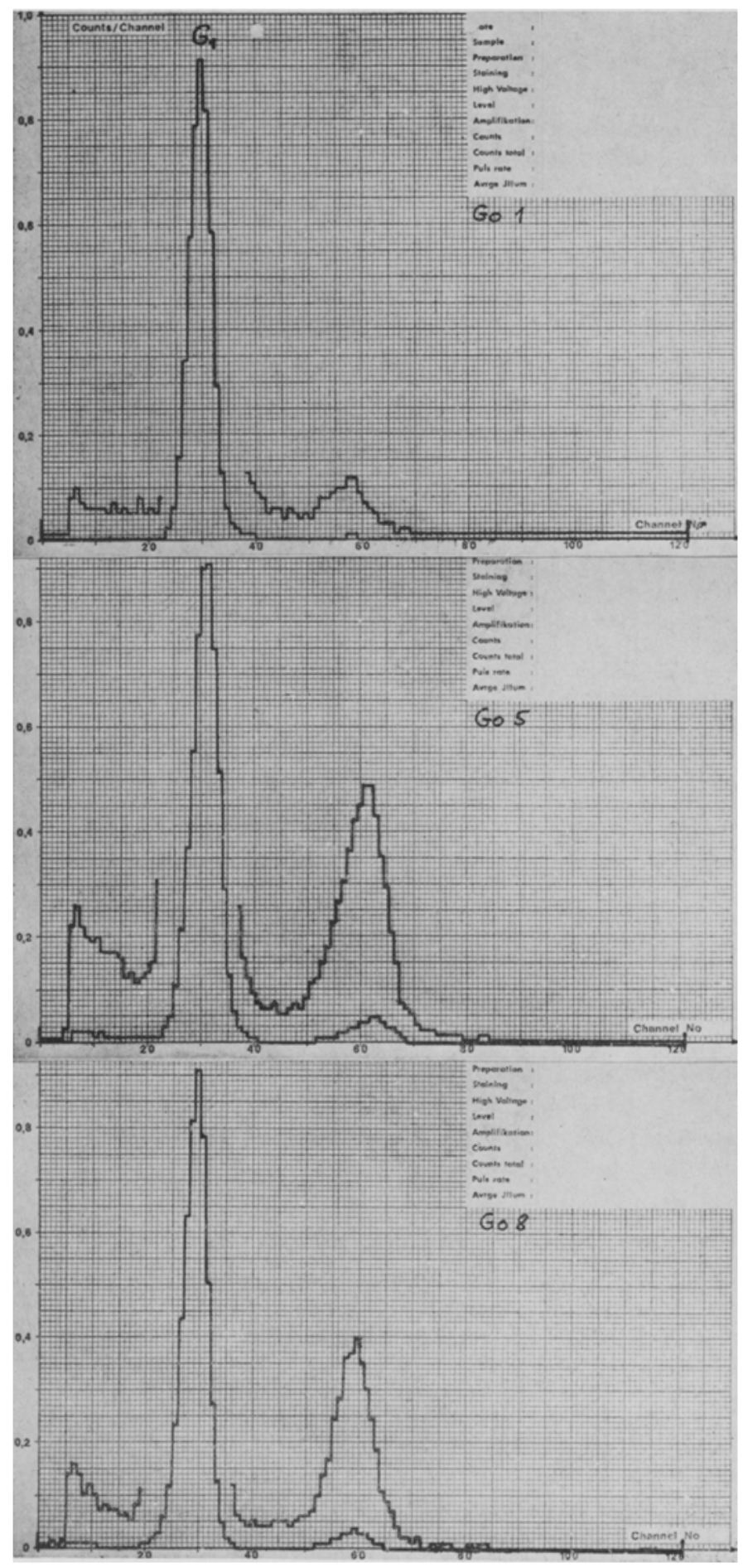


der klinischen Besserung abnahm (Abb. 1). Stellt sich der Interpretation von DNAHistogrammen aus dem Knochenmark die Schwierigkeit entgegen, daß es sich außer im Schub der akuten Leukämie mit nahezu 100\% Blasten meist um eine sehr heterogene Zellpopulation aus den verschiedenen normalen und pathologischen Zellformen handelt, so sind die Verhältnisse im peripheren Blut klarer, da hier praktisch nur Zellen der Leukämie oder lymphatische Reizformen bei Virusinfekt Proliferation bzw. erhöhte DNA-Werte aufweisen.

Aneuploide Zellinien konnten bei einzelnen Leukämien auch ohne ablaufende Mitosen anhand von Extragipfeln neben dem $G_{1}-$ Gipfel wahrscheinlich gemacht werden (Büchner et al. 1972 [3]).

Der vielleicht größte Nutzen der ICP für die klinische Hämatologie zeichnet sich jedoch jetzt zunehmend in der Möglichkeit ab, zytozide und zellkinetische Effekte der Leukämiebehandlung im Histogramm zu erkennen und ständig zu verfolgen. So scheint ein günstigeres Prognostikum für das Ansprechen auf die Chemotherapie in einem höheren Anteil proliferierender Zellen im Knochenmark bei akuten Leukämien zu liegen (Hillen und Haanen 1973 [10]). Unter Chemotherapie interessieren besonders Zeichen der Zellschädigung in Form vermehrter Zellfragmente (Abb. 2) und Zeichen der Arretierung von Zellen in bestimmten Phasen der Proliferation. So entwickelte sich unter Vincristin im Knochenmark eine Zunahme von Zellen mit doppeltem DNA-Wert auf das 4 bis 5 fache durch Blockierung in der Mitose (Büchner et al. 1972 [3]).

Selbst im peripheren Blut, das für die Therapiekontrolle leicht zu gewinnen ist, zeigten sich unter Chemotherapie akuter Leukämic Anreicherungen von Zellen in bestimmten Phasen, so in der frühen S-Phase unter Cytosin-arabinosid und Adriamycin (Abb. 3, S. 4), oder über den gesamten S-Phasenbereich verteilt unter Cytosin-arabinosid (Büchner et al. 1973c [6a]) und schließlich am $\mathrm{G}_{2}$-Gipfel nach Dauno-Rubidomycin plus Vincristin (Abb. 4, S. 5). Arretierungseffekte ähnlicher Form teilten auch Hillen und Haanen (1973 [10]) sowie Maj et al. (1973 [13]) mit.

In vorliegender Studie erreichte die Akkumulation von Zellen im frühen SPhasebereich teilweise einen solchen Grad, daß eine echte Zunahme proliferierender Zellen zu diskutieren war etwa durch Aktivierung von $\mathrm{G}_{0}-$ Zellen oder Ausschwemmung von Zellen im Proliferationszyklus aus dem Knochenmark unter Wirkung der Chemotherapie.

Bei den beschriebenen Akkumulationseffekten im DNA-Histogranm des Blutes handelt es sich um Erscheinungen, die nur in vivo unter Chemotherapie von Leukämien auftraten und durch Inkubation mit 100facher therapeutischer Konzentration der entsprechenden Zytostatika über mehrere Tage in vitro (etwa als Artefakte) nie zu beobachten waren.

Im Knochenmark ergab sich inzwischen bei Patienten mit akuten myeloischen Leukämien überwiegend eine deutliche Zunahme des S-Phaseanteils bis auf das 4 fache unter 48 stündiger Infusion von $6 \mathrm{mg} / \mathrm{kg}$ Cytosin-arabinosid (Büchner et al. $1973[66]$.

Durch derartige ständigen Einblicke in die zellkinetischen Auswirkungen der Chemotherapie scheint sich ein neuer Weg zu eröffnen, wie Zytostatika zeitlich gezielt und individuell als tatsächliche Synchronisationstherapie kombiniert werden können. 
Inzwischen hat eine Reihe von Autoren die Impulszytophotometrie in der Hämatologie erprobt (Müller et al. 1972 [14], Hillen und Haanen 1973 [10], Lang et al. 1973 [11], Maj et al. 1973 [13], Senn et al. 1973 [15] und Smets 1973 [16]). Kritische Anmerkungen bezogen sich vor allem auf die Möglichkeit von Zellverklebungen bei der Präparation und auf eine geringe Empfindlichkeit und Genauigkeit bei der Etfassung kleiner Anteile von S-Phase-Zellen (Müller et al. 1972 [14]), Fehler, die durch adäquate Vorbehandlung der Zellen (Büchner et al. 1973 [5]) zu vermeiden sein müßten.

Literatur: Büchner, Th., W. Dittrich und W. Göhde: Die Impulscytophotometrie in der hämatologischen Cytologie. Klin. Wschr. 49, 1090 (1971). - 2 Büchner, Th., W. Dittrich und W. Göhde: Impulscytophotometrie von Blut- und Knochenmarkszellen. Verh. Dtsch. Ges. Inn. Med. 77, 416 (1971). - 3 Büchner, Th., W. Dittrich und W. Göhde: Automatische DNA-Messungen zur Zellkinetik, von Leukämien mit Hilfe der Impulscytophotometrie. In: Leukämie, Herausgeber R. Groß und J. van de Loo, Springer-Verlag 1972. 4 Büchner, Th., W. Hiddemann, R. Schneider, D. Kamanabroo und W. Göhde: Untersuchungen über zellkinetische Effekte der Leukämiebehandlung in der Klinik anhand des DNS-Histogramms mittels Impulscytophotometrie. Med. Welt 24, 1616 (1973). 5 Büchner, Th., W. Hiddemann und R. Schneider: Zur Präparation von Blut- und Knochenmarkszellen für die Impulscytophotometrie. In: Symposion Impulscytophotometrie, Springer-Verlag 1973 (im Druck). — 6a Büchner, Th., W. Göhde, R. Schneider, W. Hiddemann und.D. Kamanabroo: Zellsynchronisation und cytocide Effekte durch Chemotherapie der Leukämie in der Klinik anhand der Impulscytophotometrie. In: Symposion Impulscytophotometrie. Springer-Verlag 1973 (im Druck). - 6b Büchner, Th., B. Barlogie, V. Asseburg, W. Widdernann, D. Kamanabroo und W. Göhde: Accumulation of S-phase cells by Cytosine arabinoside in the bone marrow of Patients with acute leukemia. Blut (im Druck, 1973d). - 7 Dittrich, W. und W. Göhde: Impulsfluorometrie bei Einzelzellen in Suspension. Z. Naturforsch. 246, 360 (1969). - 8 Göhde, W.: Automation in der quantitativen Cytologie mit der Impulscytophotometrie. GBK-Mitteil. 6, 255 (1972). 9 Göhde, W.: Zellcyklusanalysen mit dem Impulscytophotometer. Habilitationsschrift Univ. Münster 1973. - 10 Hillen, H. und C. Haanen: Impulscytophotometrie von Knochenmarkszellen bei akuter Leukämie. In: Symposion Impulscytophotometrie, Springer-Verlag 1973 (im Druck). - 11 .Lang, H. J., E. Feil und H. J. Senn: Zur Methodik der impulscytophotometrischen DNS-Bestimmung in normalen und pathologischen Zellpopulationen. In: Symposion Impulscytophotometrie, Springer-Verlag 1973 (im Druck). - 12 LePecq, J. B. und C. Paoletti: A fluorescent complex between Ethidium bromide and nucleic acids. J. molec. Biol. 27, 87 (1967). - $13 \mathrm{Maj}$, St., D. Lutz und A. Stacher: Impulscytophotometric investigations during cytostatic therapy in leukemic patients (preliminary results). In: Symposion Impulscytophotometrie, Springer-Verlag 1973 (im Druck). - 14 Müller, D., E. Reichert, H. D. Lang, A. Simon und H. Ch. Benöhr: Die Möglichkeiten der Impulscytophotometrie für die Bestimmung der Zellproliferation bei Hämoblastosen. In: Leukämie, Hrsgb. R. Gross und J. van de Loo, Springer-Verlag 1972. - 15 Senn, H. J., H. J. Lang und E. Feil: Impulscytophotometrische Charakterisierung der DNS-Verteilung in malignen Ergüssen und soliden Tumoren. In: Symposion Impulscytophotometrie, Springer-Verlag 1973 (im Druck). - 16 Smets, L. A.: Binding of Acridine Orange in resting and stimulated cells. In: Symposion Impulscytophotometrie, SpringerVerlag 1973 (im Druck).

Anschr. d. Verf.: Prof. Dr. Th. Büchner, Med. Klinik und Poliklinik d. Univ. Münster, D-44 Münster/Westf. 